\title{
Mean platelet volume in bipolar disorder: the search for an ideal biomarker
}

\author{
This article was published in the following Dove Press journal: \\ Neuropsychiatric Disease and Treatment \\ 16 August 2016 \\ Number of times this article has been viewed
}

\author{
Derya Guliz Mert' \\ Hatice Terzi \\ 'Department of Psychiatry, \\ ${ }^{2}$ Department of Hematology, Faculty \\ of Medicine, Cumhuriyet University, \\ Sivas, Turkey
}

\begin{abstract}
Background: The pathophysiology of bipolar disorder (BD) remains a mystery. In this context, interest in the role of the immune and inflammatory systems in BD has been increasing. We aimed to compare the routine hemogram values of BD patients with those of the participants in the healthy control group, to assess the inflammation levels of the two groups. Mean platelet volume (MPV) can be obtained as routine hemogram parameters and may aid in the detection of systemic inflammation.
\end{abstract}

Subjects and methods: This study was conducted with BD (manic episode) inpatients ( $\mathrm{n}=132$ ) and healthy controls $(n=135)$. Abnormally distributed variables (ie, neutrophil-lymphocyte ratio [NLR], platelet-lymphocyte ratio [PLR], neutrophils, lymphocytes, hemoglobin, hematocrit [HCT], mean corpuscular volume [MCV], mean corpuscular hemoglobin [MCH], mean corpuscular hemoglobin concentration [MCHC], red cell distribution width [RDW], MPV, and plateletcrit [PCT]) were compared using the Mann-Whitney $U$-test. Student's $t$-test was used to compare the mean ages and white blood cell, red blood cell, and platelet counts of the patients with $\mathrm{BD}$ against those of the participants in the control group.

Results: The comparisons revealed that while the mean WBC and the median NLR, PLR, neutrophil, lymphocyte, MPV, and PCT values were significantly higher in the patients with $\mathrm{BD}(P<0.05)$, the median hemoglobin, $\mathrm{RBC}, \mathrm{HCT}$, and $\mathrm{MCHC}$ values were significantly higher in the control group $(P<0.05)$.

Conclusion: Comparisons of hemogram values of patients with $\mathrm{BD}$ against those of the healthy control group revealed that inflammatory cells (absolute neutrophil count, platelet count, PCT, and MPV) and ratios (NLR, PLR) seem to be altered during manic episodes. These findings support the hypothesis that inflammatory activation occurs in BD during manic episodes. In addition to NLR and PLR, MPV may be useful in the detection of this activation. The most significant limitation in the study is that smokers were not excluded in both groups. The development of new preventive and therapeutic options can be facilitated through the understanding of this mechanism because through this mechanism, inflammation may pathologically affect brain function, as well as inducing and/or perpetuating BD.

Keywords: bipolar disorder, inflammation, mean platelet volume

\section{Introduction}

Bipolar disorder (BD) is a chronic, severe, and highly disabling illness. ${ }^{1}$ The pathophysiology of BD is still not thoroughly obvious. In this context, the roles of the immune and inflammatory systems in BD have attracted increasing attention. ${ }^{2,3}$ Mood episodes have been demonstrated to be associated with changes in cytokine profiles, ${ }^{4-6}$ levels of acute phase proteins such as immunoglobulins, complement proteins, and factor B, ${ }^{7}$ high-sensitivity C-reactive protein ${ }^{8}$ ratio of the absolute neutrophil-to-lymphocyte counts (NLR), and ratio of the absolute platelet-to-lymphocyte counts (PLR). ${ }^{9}$
Correspondence: Derya Guliz Mert Department of Psychiatry, Faculty of Medicine, Cumhuriyet University, 58140 Sivas, Turkey Tel +903462580000 Emaildrgulizaksoy@yahoo.com (c)
hereby accept the Terms. Non-commercial uses of the work are permitted without any further permission from Dove Medical Press Limited, provided the work is properly attributed. For permission hereby accept the Terms. Non-commercial uses of the work are permitted without any further permission from Dove Medions.
for commercial use of this work, please see paragraphs 4.2 and 5 of our Terms (https://www.dovepress.com/terms.php). 
In clinical practice, it has been reported that hemogram parameters such as the mean platelet volume (MPV), NLR, and PLR can be used as markers of systemic inflammation in different diseases. ${ }^{10-12}$ The MPV level can be determined during routine blood counts, but unfortunately, clinicians do not usually pay much attention to this parameter. Platelet volume is determined based on the number of megakaryocytes present during platelet production, and this factor is associated with platelet function and activation. Normally, the size and number of platelets are inversely correlated. ${ }^{13,14}$ The MPV has been studied as a simple inflammatory marker in several diseases. NLR and PLR are among the laboratory markers that have been introduced into clinical practice for evaluation of systemic inflammation. ${ }^{15}$ NLR, PLR, and MPV have been defined as novel potential markers for evaluating inflammation in various diseases, including oncologic diseases, cardiovascular diseases (eg, heart failure, hypertension, acute coronary syndromes, coronary artery disease, myocardial infarction, ST-segment elevation, and coronary revascularization procedures), cerebrovascular diseases (eg, cerebrovascular events, Alzheimer's disease, and Parkinson's disease), end-stage renal disease, and inflammatory diseases (eg, ankylosing spondylitis, ulcerative colitis, appendicitis, and pelvic inflammatory disease). ${ }^{10-12,16}$

There has been growing interest in several immune and inflammatory alterations in BD. ${ }^{17,18}$ Other tests for markers of systemic inflammation that are described in the literature are costly and require better-equipped laboratories. ${ }^{19-21}$ However, MPV was evaluated in this study; it can be determined under simple laboratory conditions and assessed using easily accessible and inexpensive tests. MPV can be obtained as routine hemogram parameters and may thus aid in the detection of systemic inflammation. The values obtained from hemogram parameters are practical and can reduce the extra economic burden that arises from additional tests. In a recent study, NLR and PLR were investigated and found to be significantly elevated in BD patients. ${ }^{9}$ Our search for studies in which MPV was used as an inflammation marker in BD patients demonstrated a gap in the literature. Although a satisfactory biological explanation for $\mathrm{BD}$ is not currently available, the role of systemic inflammation in the pathophysiology of BD has been studied in the past decade with increasing interest. Therefore, we aimed to compare the routine hemogram values, including MPV, of BD patients with those of participants in a healthy control group to assess the inflammation levels of these groups.

\section{Subjects and methods Patients}

The medical and clinical psychiatric records of all of the BD inpatients $(\mathrm{n}=172)$ (based on the criteria in Diagnostic and Statistical Manual of Mental Disorders, Fourth Edition) admitted to the psychiatry service of Cumhuriyet University Hospital, Turkey, between 2012 and 2014 were retrospectively examined. Patients with manic episodes during admission were included. The participants were selected from among the manic patients who were drug free for at least 2 weeks. ${ }^{9}$ At the end of the screening of the medical and clinical psychiatric records, patients with alcohol and/or substance dependence, comorbid axis 1 disorders, and/or other medical diseases (eg, acute or chronic endocrinological, inflammatory, or autoimmune diseases) and those who were suspected to have used psychiatric drugs before admission were not included. After considering all exclusion criteria, 132 BD manic episode patients were included in the study.

\section{Healthy controls}

Age- and sex-matched control subjects $(n=135)$ who were recruited in other clinics of Cumhuriyet University Hospital, Turkey, were included. None of the controls was taking any form of prescribed psychiatric medication. None of the healthy volunteers met the exclusion criteria listed earlier. The information regarding the exclusion criteria and the use of any form of prescribed psychiatric medication was supported by data gathered from the participants, their firstdegree relatives, and the participants' files.

Before beginning the study, approval was obtained from the Human Research Ethics Committee of Cumhuriyet University Hospital. Written informed consent was obtained from all the participants.

\section{Hematologic examinations}

The patients' hemogram values were determined on the first day of their hospitalization in the psychiatric clinic (including white blood cell [WBC], neutrophil, lymphocyte, platelet, and red blood cell $[\mathrm{RBC}]$ counts, hemoglobin, hematocrit [HCT], mean corpuscular volume [MCV], mean corpuscular hemoglobin $[\mathrm{MCH}]$, mean corpuscular hemoglobin concentration $[\mathrm{MCHC}]$, red cell distribution width [RDW], MPV, and plateletcrit $[\mathrm{PCT}]$ ) and these were obtained from the hospital's archives. The NLR and the PLR were calculated. In the inpatient psychiatry clinic, the hemogram measurements were performed using the peripheral venous blood samples obtained upon admission. The blood samples were collected 
into tubes containing EDTA. A fully automated blood cell counter (BC-6800; Mindray, Shenzhen, People's Republic of China) was used for the hemogram measurements.

\section{Statistics}

Descriptive statistics were used to summarize all of the variables. The data are expressed as the mean $\pm \mathrm{SD}$ values or percentages as appropriate. Normality was tested using the Kolmogorov-Smirnov test. Abnormally distributed variables (ie, NLR, PLR, neutrophils, lymphocytes, hemoglobin, HCT, $\mathrm{MCV}, \mathrm{MCH}, \mathrm{MCHC}, \mathrm{RDW}, \mathrm{MPV}$, and PCT) were compared using the Mann-Whitney $U$-test. Student's $t$-test was used to compare the mean ages and the $\mathrm{WBC}, \mathrm{RBC}$, and platelet counts of the patients with BD against those of the participants in the control group. The sex ratios of the study groups were compared using the chi-square $\left(\chi^{2}\right)$ test. The Statistical Package for the Social Sciences version 22 (IBM Corporation, Armonk, NY, USA) for Windows, version 22.0, was used to analyze the data. $P<0.05$ was regarded as significant.

\section{Results}

In this study, 132 patients were included. There were no differences between the patients and the control group participants in terms of age or sex. The mean ages of the patients and the control group participants were $40.2 \pm 12.2$ years and $40.1 \pm 12.1$ years, respectively. Sixty-six $(50.0 \%)$ of the patients and $69(51.1 \%)$ of the control group participants were female (Table 1).

The laboratory findings for the two study groups are presented in Table 2. The mean WBC count and the median NLR, PLR, neutrophil, lymphocyte, MPV, and PCT values of the patients with $\mathrm{BD}$ were significantly greater than those of the control group $(P<0.05)$. The median hemoglobin, $\mathrm{RBC}$, $\mathrm{HCT}$, and MCHC values of the control group were significantly higher than those of the patients with $\mathrm{BD}(P<0.05)$. There were no differences between the two groups in terms of the MCV, MCH, RDW, or platelet values $(P>0.05)$.

Table I Characteristics of patients with bipolar disorder and the control group participants

\begin{tabular}{llll}
\hline Characteristics & $\begin{array}{l}\text { Patients } \\
(\mathbf{N}=132)\end{array}$ & $\begin{array}{l}\text { Controls } \\
(\mathbf{N}=135)\end{array}$ & P-value \\
\hline Age, years & $40.2 \pm 12.2$ & $40.1 \pm 12.1$ & 1.000 \\
Sex & & & 0.903 \\
$\quad$ Male, n (\%) & $66(50.0 \%)$ & $66(48.9 \%)$ & \\
$\quad$ Female, n (\%) & $66(50.0 \%)$ & $69(51.1 \%)$ & \\
Duration of disease, years & $14.17 \pm 9.62$ & - & \\
\hline
\end{tabular}

Notes: The data are presented as the mean \pm standard deviation and percentages. The data were compared using Student's $t$-test and the chi-square $\left(\chi^{2}\right)$ test.
Table 2 Laboratory findings for the patients with bipolar disorder and control group participants

\begin{tabular}{|c|c|c|c|}
\hline Variables & $\begin{array}{l}\text { Patient } \\
(\mathrm{N}=132)\end{array}$ & $\begin{array}{l}\text { Control } \\
(\mathrm{N}=135)\end{array}$ & $P$-value \\
\hline NLR & $2.80 \pm 1.67$ & $1.75 \pm 0.44$ & $0.00 I^{*}$ \\
\hline PLR & II $3.32 \pm 53.97$ & I I $0.57 \pm 26.99$ & $0.001 *$ \\
\hline WBCs $\left(10^{3} / \mu \mathrm{L}\right)$ & $8.16 \pm 2.31$ & $6.96 \pm 1.28$ & $0.00 I^{* *}$ \\
\hline Neutrophils $\left(10^{3} / \mu \mathrm{L}\right)$ & $5.35 \pm 2.10$ & $4.03 \pm 0.84$ & $0.00 I^{*}$ \\
\hline Lymphocytes $\left(10^{3} / \mu \mathrm{L}\right)$ & $2.19 \pm 0.78$ & $2.36 \pm 0.46$ & $0.003 *$ \\
\hline Hemoglobin (g/dL) & $|4.15 \pm 1.8|$ & $15.00 \pm 1.19$ & $0.00 I^{*}$ \\
\hline RBCs $\left(10^{6} / \mu \mathrm{L}\right)$ & $4.88 \pm 0.5 \mid$ & $5.06 \pm 0.41$ & $0.00 I^{* *}$ \\
\hline HCT (\%) & $42.65 \pm 5.59$ & $43.73 \pm 4.65$ & $0.008^{*}$ \\
\hline MCV (fL) & $86.92 \pm 5.81$ & $86.56 \pm 3.22$ & $0.072^{*}$ \\
\hline $\mathrm{MCH}(\mathrm{pg})$ & $29.03 \pm 2.52$ & $29.55 \pm 1.32$ & $0.426 *$ \\
\hline $\mathrm{MCHC}(\mathrm{g} / \mathrm{dL})$ & $33.36 \pm 1.3$ & $34.0 I \pm 0.78$ & $0.001 *$ \\
\hline RDW (\%) & $13.75 \pm 1.62$ & $13.27 \pm 0.58$ & $0.37 I^{*}$ \\
\hline Platelets $\left(10^{3} / \mu \mathrm{L}\right)$ & $263.55 \pm 60.69$ & $253.33 \pm 51.27$ & $0.11 I^{* *}$ \\
\hline $\mathrm{MPV}(\mathrm{fL})$ & $9.43 \pm 1.03$ & $9.11 \pm 0.83$ & $0.034^{*}$ \\
\hline PCT (\%) & $0.25 \pm 0.05$ & $0.23 \pm 0.06$ & $0.03 I^{*}$ \\
\hline
\end{tabular}

Notes: The data are expressed as the mean \pm standard deviation. *Mann-Whitney U-test. **Student's $t$-test.

Abbreviations: $\mathrm{HCT}$, hematocrit; $\mathrm{MCH}$, mean corpuscular hemoglobin; $\mathrm{MCHC}$, mean corpuscular hemoglobin concentration; MCV, mean corpuscular volume; MPV, mean platelet volume; NLR, neutrophil-to-lymphocyte ratio; PCT, plateletcrit; PLR, platelet-to-lymphocyte ratio; RBC, red blood cell; RDW, red cell distribution width; WBC, white blood cell.

\section{Discussion}

We attempted to evaluate the inflammation status of patients with BD by comparing the hemogram parameters between patients with $\mathrm{BD}$ and healthy control group participants. This study revealed that the NLR, PLR, MPV, absolute neutrophil count, platelet counts, and PCT in patients with BD were higher than those in the healthy control group participants. However, hemoglobin and other erythrocyte indexes were significantly lower in the experimental group than in the control group participants.

Inflammatory responses resulting from infection, cellular damage, or stress can be considered appropriate, physiologic, and necessary. ${ }^{22}$ In contrast, such responses can be considered inappropriate, pathologic, and damaging when they are excessively elicited by a given stimulus or elicited by the wrong stimuli. Such inappropriate responses lead to unwanted and unwarranted effects. The harmful effects of inflammation include alterations in cognition, sleep, mood, energy, and motivation, and all such alterations are included in the symptomatology of mood disorders. ${ }^{23}$ The role of systemic inflammation in the pathophysiology of BD is not thoroughly understood. Yet, studies have shown that psychiatric symptoms in animal models and humans can be caused by peripheral immune modulators. ${ }^{24-28}$ Injection of 
proinflammatory cytokines interleukin- $1 \beta$ and tumor necrosis factor alpha into healthy animals leads to the animals showing sickness behavior related with solitude. ${ }^{25}$ Medical conditions linked with diabetes, obesity, rheumatoid arthritis, malignancies, and multiple sclerosis among the chronic inflammatory and immunological abnormalities are indicated as risk factors for depression and BD. ${ }^{24-26,28,29}$ The presence of a positive correlation between these conditions and psychiatric disease indicates that there is a prevalent inherent inflammatory process influencing the brain among other organs. ${ }^{24-26,28-31}$ Immune signals are transferred from the periphery to the brain through various existing pathways. ${ }^{25}$ The effects of cytokines on the production and degradation of serotonin are significant in the brain as well. Moreover, evidence suggests that cytokines activate the hypothalamic-pituitary-adrenal axis and induce glucocorticoid insensitivity. ${ }^{32}$ Another significant role played by microglial cells in terms of pathologic synaptic pruning may cause maladaptive brain function. ${ }^{33}$ Finally, impaired neuroplasticity, which leads to functional and structural central nervous system changes and which is induced by inflammation, may also be implicated. ${ }^{22,30}$

According to some investigators, elevations in inflammatory markers may indicate acute changes during depressive and manic episodes; serum marker concentrations peak above the baseline during mood episodes and drop during euthymic states. ${ }^{34}$ Similar findings have repeatedly been reported by several investigators, who have observed increased incidences of mood symptoms and mood episodes in BD patients with elevated levels of inflammatory markers in the peripheral blood and cerebrospinal fluid. ${ }^{4,35-39}$ NLR and PLR have been indicated to be markers of inflammation in many studies, and in this study, these ratios were found to be elevated during manic episodes in patients with BD. These findings support the results of a study by Kalelioglu et al. ${ }^{9}$ Additionally, in this study, the WBC, neutrophil, and lymphocyte counts were found to be higher in BD patients than in the healthy controls, which supports the presence of inflammation in BD patients. MPV levels have been found to be elevated in various diseases characterized by inflammation; ${ }^{12,40}$ therefore, MPV has been regarded as an inflammation marker. In previous studies, no relationships were observed between the MPV levels of BD patients and inflammation; however, in this study, the MPV levels of BD patients were found to be elevated. This result suggests that, similar to NLR and PLR, the MPV value could be used as a marker of inflammation in patients with BD. Moreover, the hemoglobin, RBC, HCT, and $\mathrm{MCHC}$ levels of the $\mathrm{BD}$ patients were lower than those of the control group participants. We believe that inflammation plays a very powerful role in the etiopathogenesis of BD and that many of the inflammatory cytokines in the systemic circulation suppress erythropoiesis; consequently, these patients develop anemia of inflammation, which is also known as anemia of chronic disease. ${ }^{41}$

This study has some limitations. First, because the study was retrospective, there were limitations regarding the evaluated data. The first such limitation is that the participants' body mass indexes and smoking statuses were not known. Because these data were not included in the document files and archives, obese individuals and smokers were not excluded from the study. Because obesity and smoking may cause inflammation, ${ }^{42,43}$ these factors may have increased the amount of inflammation in the subjects included in the study. Additional limitations due to the retrospective design of the study are that the diagnoses were obtained from the files in the archives and not through face-to-face interviews and that the severities of the manic episodes were not assessed. This limitation is particularly relevant because correlations have been established between symptom severity and inflammation in some studies. ${ }^{42,43}$ With more detailed and prospective studies, the prognostic importance of inflammation can be demonstrated. The exclusion of patients in the euthymic phase from the study represents another limitation. An additional limitation is that immune system indicators such as cytokines were not evaluated; therefore, it is not possible to determine whether increased MPV is an independent marker of alterations in the immune system in patients with BD.

\section{Conclusion}

The results presented herein clearly demonstrate that the MPV, NLR, and PLR values of the patients with BD were higher than those of the healthy participants in the control group. A comparison of the hemogram values between the patients with $\mathrm{BD}$ and the healthy subjects of the control group demonstrated that inflammatory cells and their ratios seem to be altered during manic episodes. This finding supports the hypothesis that an inflammatory activation occurs in BD patients during manic episodes. Similarly, NLR, PLR, and MPV may be useful for detecting this activation. The development of new preventive and therapeutic options can be expedited through the understanding of this mechanism because it is through this mechanism that inflammation may pathologically affect brain function and thereby induce and/ or perpetuate $\mathrm{BD} .^{22}$

\section{Disclosure}

The authors report no conflicts of interest in this work. 


\section{References}

1. Belmaker RH. Bipolar disorder. N Engl J Med. 2004;351(5):476-486.

2. Fiedorowicz JG, Prossin AR, Johnson CP, Christensen GE, Magnotta VA, Wemmie JA. Peripheral inflammation during abnormal mood states in bipolar I disorder. J Affect Disord. 2015;187:172-178.

3. Uyanik V, Tuglu C, Gorgulu Y, Kunduracilar H, Uyanik MS. Assessment of cytokine levels and hs-CRP in bipolar I disorder before and after treatment. Psychiatry Res. 2015;228(3):386-392.

4. Kim YK, Jung HG, Myint AM, Kim H, Park SH. Imbalance between pro-inflammatory and anti-inflammatory cytokines in bipolar disorder. $J$ Affect Disord. 2007;104(1-3):91-95.

5. Newcomer JW. Medical risk in patients with bipolar disorder and schizophrenia. J Clin Psychiatry. 2006;67(11):e16.

6. O'Brien SM, Scully P, Scott LV, Dinan TG. Cytokine profiles in bipolar affective disorder: focus on acutely ill patients. $J$ Affect Disord. 2006;90(2-3):263-267.

7. Wadee AA, Kuschke RH, Wood LA, Berk M, Ichim L, Maes M. Serological observations in patients suffering from acute manic episodes. Hum Psychopharmacol. 2002;17(4):175-179.

8. Hornig M, Goodman DB, Kamoun M, Amsterdam JD. Positive and negative acute phase proteins in affective subtypes. $J$ Affect Disord. 1998;49(1):9-18.

9. Kalelioglu T, Akkus M, Karamustafalioglu N, et al. Neutrophillymphocyte and platelet-lymphocyte ratios as inflammation markers for bipolar disorder. Psychiatry Res. 2015;228(3):925-927.

10. Kuyumcu ME, Yesil Y, Ozturk ZA, et al. The evaluation of neutrophillymphocyte ratio in Alzheimer's disease. Dement Geriatr Cogn Disord. 2012;34(2):69-74

11. Boyraz I, Koç B, Boyacı A, Tutoğlu A, Sarman H, Ozkan H. Ratio of neutrophil/lymphocyte and platelet/lymphocyte in patient with ankylosing spondylitis that are treating with anti-TNF. Int J Clin Exp Med. 2014;7(9):2912-2915.

12. Kilincalp S, Coban S, Akinci H, et al. Neutrophil/lymphocyte ratio, platelet/lymphocyte ratio, and mean platelet volume as potential biomarkers for early detection and monitoring of colorectal adenocarcinoma. Eur J Cancer Prev. 2015;24(4):328-333.

13. Jackson SR, Carter JM. Platelet volume: laboratory measurement and clinical application. Blood Rev. 1993;7(2):104-113.

14. Threatte GA. Usefulness of the mean platelet volume. Clin Lab Med. 1993;13(4):937-950.

15. Balta S, Demirkol S, Kucuk U. The platelet lymphocyte ratio may be useful inflammatory indicator in clinical practice. Hemodial Int. 2013;17(4):668-669.

16. Kopuz A, Turan V, Ozcan A, et al. A novel marker for the assesment of the treatment result in pelvic inflammatory disease. Minerva Ginecol. Epub 2014 Oct 17.

17. RosenblatJD, BrietzkeE, MansurRB, MaruschakNA, Lee Y,McIntyreRS. Inflammation as a neurobiological substrate of cognitive impairment in bipolar disorder: evidence, pathophysiology and treatment implications. $J$ Affect Disord. 2015;188:149-159.

18. Anderson G, Maes M. Bipolar disorder: role of immune-inflammatory cytokines, oxidative and nitrosative stress and tryptophan catabolites. Curr Psychiatry Rep. 2015;17(2):8.

19. Reichenberg A, Yirmiya R, Schuld A, et al. Cytokine-associated emotional and cognitive disturbances in humans. Arch Gen Psychiatry. 2001;58(5):445-452.

20. Wright CE, Strike PC, Brydon L, Steptoe A. Acute inflammation and negative mood: mediation by cytokine activation. Brain Behav Immun. 2005;19(4):345-350.

21. Boufidou F, Nikolaou C, Alevizos B, Liappas IA, Christodoulou GN. Cytokine production in bipolar affective disorder patients under lithium treatment. J Affect Disord. 2004;82(2):309-313.

22. Rosenblat JD, Cha DS, Mansur RB, McIntyre RS. Inflamed moods: a review of the interactions between inflammation and mood disorders. Prog Neuropsychopharmacol Biol Psychiatry. 2014;53:23-34.
23. Miller AH, Maletic V, Raison CL. Inflammation and its discontents: the role of cytokines in the pathophysiology of major depression. Biol Psychiatry. 2009;65(9):732-741.

24. Najjar S, Pearlman DM, Alper K, Najjar A, Devinsky O. Neuroinflammation and psychiatric illness. J Neuroinflammation. 2013;10:43.

25. Dantzer R, O'Connor JC, Freund GG, Johnson RW, Kelley KW. From inflammation to sickness and depression: when the immune system subjugates the brain. Nat Rev Neurosci. 2008;9(1):46-56.

26. Laske C, Zank M, Klein R, et al. Autoantibody reactivity in serum of patients with major depression, schizophrenia and healthy controls. Psychiatry Res. 2008;158(1):83-86.

27. Benros ME, Nielsen PR, Nordentoft M, Eaton WW, Dalton SO, Mortensen PB. Autoimmune diseases and severe infections as risk factors for schizophrenia: a 30-year population-based register study. Am J Psychiatry. 2011;168(12):1303-1310.

28. Leboyer M, Soreca I, Scott J, et al. Can bipolar disorder be viewed as a multi-system inflammatory disease? J Affect Disord. 2012;141(1): $1-10$.

29. McNally L, Bhagwagar Z, Hannestad J. Inflammation, glutamate, and glia in depression: a literature review. CNS Spectr. 2008;13(6):501-510.

30. Harrison NA, Brydon L, Walker C, Gray MA, Steptoe A, Critchley HD. Inflammation causes mood changes through alterations in subgenual cingulate activity and mesolimbic connectivity. Biol Psychiatry. 2009; 66(5):407-414.

31. Haroon E, Raison CL, Miller AH. Psychoneuroimmunology meets neuropsychopharmacology: translational implications of the impact of inflammation on behavior. Neuropsychopharmacology. 2012; 37(1):137-162.

32. Wang J, Dunn AJ. Mouse interleukin-6 stimulates the HPA axis and increases brain tryptophan and serotonin metabolism. Neurochem Int. 1998;33(2):143-154.

33. Stertz L, Magalhaes PV, Kapczinski F. Is bipolar disorder an inflammatory condition? The relevance of microglial activation. Curr Opin Psychiatry. 2013;26(1):19-26.

34. Remlinger-Molenda A, Wójciak P, Michalak M, et al. Activity of selected cytokines in bipolar patients during manic and depressive episodes. Psychiatr Pol. 2012;46(4):599-611.

35. Breunis MN, Kupka RW, Nolen WA, et al. High numbers of circulating activated $\mathrm{T}$ cells and raised levels of serum IL-2 receptor in bipolar disorder. Biol Psychiatry. 2003;53(2):157-165.

36. Cunha AB, Andreazza AC, Gomes FA, et al. Investigation of serum high-sensitive C-reactive protein levels across all mood states in bipolar disorder. Eur Arch Psychiatry Clin Neurosci. 2008;258(5):300-304.

37. Tsai SY, Chen KP, Yang YY, et al. Activation of indices of cell-mediated immunity in bipolar mania. Biol Psychiatry. 1999;45(8):989-994.

38. Stich O, Andres TA, Gross CM, Gerber SI, Rauer S, Langosch JM. An observational study of inflammation in the central nervous system in patients with bipolar disorder. Bipolar Disord. 2015;17(3):291-302.

39. Rolstad S, Jakobsson J, Sellgren C, et al. CSF neuroinflammatory biomarkers in bipolar disorder are associated with cognitive impairment. Eur Neuropsychopharmacol. 2015;25(8):1091-1098.

40. Topal E, Celiksoy MH, Catal F, Karakoç HT, Karadağ A, Sancak R. The platelet parameters as inflammatory markers in preschool children with atopic eczema. Clin Lab. 2015;61(5-6):493-496.

41. Theurl I, Mattle V, Seifert M, Mariani M, Marth C, Weiss G. Dysregulated monocyte iron homeostasis and erythropoietin formation in patients with anemia of chronic disease. Blood. 2006;107(10):4142-4148.

42. Wang Y, Huang F. N-3 polyunsaturated fatty acids and inflammation in obesity: local effect and systemic benefit. Biomed Res Int. 2015;2015: 581469.

43. Daloee MH, Avan A, Mirhafez SR, et al. Impact of cigarette smoking on serum pro- and anti-inflammatory cytokines and growth factors. Am J Mens Health. Epub 2015 Sep 7. 


\section{Publish your work in this journal}

Neuropsychiatric Disease and Treatment is an international, peerreviewed journal of clinical therapeutics and pharmacology focusing on concise rapid reporting of clinical or pre-clinical studies on a range of neuropsychiatric and neurological disorders. This journal is indexed on PubMed Central, the 'PsycINFO' database and CAS,

and is the official journal of The International Neuropsychiatric Association (INA). The manuscript management system is completely online and includes a very quick and fair peer-review system, which is all easy to use. Visit http://www.dovepress.com/testimonials.php to read real quotes from published authors.

Submit your manuscript here: http://www.dovepress.com/neuropsychiatric-disease-and-treatment-journal 